\title{
Antecedent clinical features associated with dementia in Parkinson's disease
}

\author{
Yaakov Stern, PhD; Karen Marder, MD, MPH; Ming Xi Tang, PhD; and Richard Mayeux, MD
}

\begin{abstract}
Article abstract-In a prospective cohort study, we examined clinical features in 250 nondemented patients with idiopathic Parkinson's disease (PD) and then evaluated their association with incident dementia during 5 years of follow-up. Seventy-four of the patients became demented. Odds ratios for incident dementia with PD were increased for the following: being older than 70 years of age $(2.7 ; 1.4$ to 5.5$)$, having a PD rating scale score greater than $25(3.0 ; 1.5$ to 6.2 ), being depressed ( $2.7 ; 1.5$ to 6.6 ), being confused or psychotic on levodopa ( $3.3 ; 1.3$ to 8.7 ), or having facial masking as a presenting sign $(6.1 ; 1.4$ to 26.9$)$.
\end{abstract}

NEUROLOGY 1993;43:1690-1692

There are few studies of the incidence of dementia in Parkinson's disease (PD) ${ }^{1-3}$ and even fewer that investigate antecedent risk factors for dementia. ${ }^{3-5}$ We have previously reported estimates of the prevalence $^{6}$ and incidence ${ }^{1}$ of dementia in a cohort of patients with PD followed at our institution, and we have explored the effects of mortality on prevalence estimates. ${ }^{7}$ In the prevalence phase of our studies, we used a data extraction form to collect information about disease onset and specific clinical features. These data are unique because they were collected prospectively, without knowledge of who would eventually become demented. We now describe our analyses to determine which, if any, of these clinical features were associated with the incident dementia during a 5-year period of follow-up.

Methods. Subjects. The flow of patients through the prevalence and incidence phases of the study is summarized in the figure. Figures differ slightly from our previous reports because of subsequent corrections due to additional chart review and further subject contact. To estimate prevalence of dementia in PD, the charts of 339 patients with idiopathic PD and age at onset greater than 40 years who had been seen as inpatients or outpatients at the Columbia-Presbyterian Medical Center between March 1, 1983, and September 30, 1984, were reviewed. Idiopathic PD was defined by the presence of two of the four cardinal features of PD conforming to standard research criteria. ${ }^{8-10}$ All subjects were seen and had their diagnosis confirmed by a neurologist. All cases of secondary or postencephalitic parkinsonism were excluded.
In this prevalence phase, 37 patients were demented.

To study incidence of dementia, charts were reviewed again 2.5 years later and the dementia status of each patient after October 1, 1984, was ascertained. Of the 302 originally nondemented patients, follow-up information was available on $250 ; 45$ had no new hospital records and could not be contacted, and a dementia diagnosis could not be ascertained in seven.

Data extraction. Prevalence period. A standard data extraction form was utilized during the prevalence period to record clinical disease features. Presence or absence of dementia and depression was determined according to DSM-III criteria. ${ }^{11}$ Dementia was defined as a loss of intellectual capacity that interfered with social or occupational function. We required either (1) a diagnosis of dementia recorded by a neurologist or psychiatrist, or (2) in the absence of a specific recorded diagnosis, recorded evidence of all signs and symptoms of dementia. For the latter, we required evidence in the records of memory loss and either aphasia, impaired judgment, loss of visuospatial skills, or an inability to perform complex mental tasks such as arithmetic. Because many of the patients at our institution had been rated on the Columbia University Parkinson's Disease Rating Scale (CUPDRS), ${ }^{12}$ we also required that the score on intellectual impairment (item 1) be two or more, indicating dementia.

We also recorded other features of PD: age at onset (defined as the first time the diagnosis of PD was made by a qualified physician); duration of PD motor manifestations (defined as the period between onset of illness and the first encounter in the study period); and cardinal motor signs present at the time of diagnosis (ie, tremor, rigidity, hypokinesia, postural instability, or masked facies). The disease was classified as with or without

From the Departments of Neurology and Psychiatry, and the Sergievsky Center, Columbia University College of Physicians and Surgeons, New York, NY.

Supported by Federal Grants AG07232 and AG08702, The Parkinson's Disease Foundation, and the Charles S. Robertson Memorial (iift for Alzheimer's Disease. 
339 patients in prevalence phase

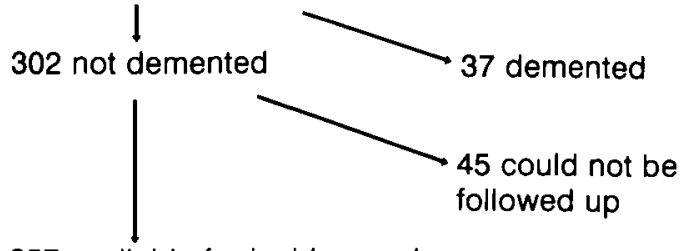

257 available for incidence phase

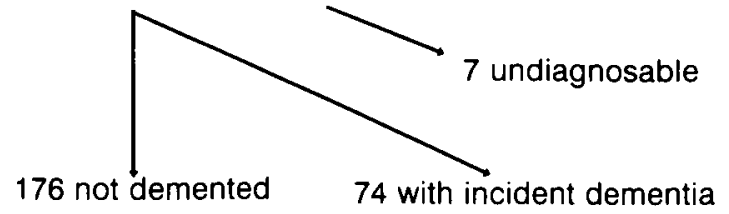

Figure. Flow of Parkinson's disease patients through the prevalence and incidence phases of the study. Antecedent clinical features were obtained for all 339 patients during the prevalence phase of the study.

resting tremor. Presence or absence of other specific motor signs at the time of evaluation was noted. In 202 patients included in the final incidence analyses, disease severity was rated using the CUPDRS. ${ }^{12}$ Response to levodopa therapy was judged by the reviewer; patients were classified as responders (good response to average doses), secondary nonresponders (good response at first, but later not adequate despite sufficient dosage), and primary nonresponders (never a good response). Adverse effects of levodopa therapy, including psychosis and confusion, were recorded. Ability to perform activities of daily living was also usually rated using the Schwab and England Activities of Daily Living Scale. ${ }^{13}$ Other disease features were also explored, including history of depression, presence or absence of depression or psychosis at the time of evaluation, and bladder control problems. The presence or absence of family history of PD in firstdegree relatives was also recorded.

The prevalence evaluation of the majority of the patients took place in their physicians' offices; 75 were hospitalized. Almost half of the data extraction forms were completed by the examining clinician at or near the time of an office visit. The other half, which included all those of hospitalized patients, were completed by chart review. The reliability of these review procedures was evaluated. ${ }^{6}$

Incidence. Two and one-half years following the prevalence study, all records were reviewed to determine the presence or absence of new dementia in the original cohort during the period October 1, 1984, to July 31, 1989. A separate data extraction form was used by neurologists to record age, sex, date of last record entry, and date when dementia was first diagnosed and recorded. Subjects not examined at the medical center after the prevalence period were telephoned. These subjects and a responsible family member were questioned using a standard interview to obtain the same information provided by the data extraction form.

The same criteria for dementia as those described for the prevalence phase were utilized. All record entries during the incidence period were reviewed, and patients were considered to have dementia only if this judgment was not contradicted by subsequent record entries.

Data analysis. For the initial analyses, subjects were divided into those who did and those who did not become
Table. Comparison of disease features ascertained during prevalence phase in patients who did and did not become demented

\begin{tabular}{|c|c|c|c|}
\hline & $\begin{array}{l}\text { Incident } \\
\text { dementia }\end{array}$ & $\begin{array}{l}\text { Not } \\
\text { demented }\end{array}$ & $p<$ \\
\hline $\mathbf{N}$ & 74 & 176 & \\
\hline \multicolumn{4}{|l|}{ At onset } \\
\hline Hypokinesia (\%) & 93.2 & 73.8 & 0.01 \\
\hline Masked facies $(\%)$ & 11.0 & 1.8 & 0.01 \\
\hline \multicolumn{4}{|l|}{ At prevalence visit } \\
\hline Age & $71.94(7.64)$ & $66.95(8.82)$ & 0.01 \\
\hline Duration of illness & $9.12(6.13)$ & $7.51(5.23)$ & 0.05 \\
\hline UPDRS score & $34.0(24.12)$ & $21.97(17.72)$ & 0.01 \\
\hline Postural instability $1 \%$ & 76.9 & 59.3 & 0.01 \\
\hline Depressed $(\%)$ & 65.7 & 40.8 & 0.01 \\
\hline Bladder control problems $(\%)$ & 14.9 & 9.1 & NS \\
\hline Psychosis from levodopa $(\%)$ & 15.3 & 3.2 & 0.01 \\
\hline Confusion from levodopa $(\%)$ & 20.8 & 7.1 & 0.01 \\
\hline $\begin{array}{l}\text { Psychosis } \\
\text { (not drug related) }(\%)\end{array}$ & 4.1 & 0.6 & 0.05 \\
\hline
\end{tabular}

Values are mean (SD) or percentages where indicated. $p$ Values are for $t$ test or chi-square comparisons as appropriate.

demented during the incidence period. Chi-square or $t$ tests were used to screen clinical disease features recorded on the data extraction form during the prevalence phase to determine possible relationships to incident dementia. Items with at least a 0.10 significance in this initial screen were then included in a logistic regression analysis, where potential antecedent clinical features were forward loaded into a model predicting incident dementia status. To aid in the interpretation of the results of the logistic regression analysis, age at onset and CUPDRS scores were each dichotomized at their respective median values. A stepwise Cox proportional hazards model with forward loading was also completed in order to take into account the prospective, temporal aspects of the study.

Results. Of the $250 \mathrm{PD}$ patients who were not demented at the close of the prevalence period for whom follow-up records were available, 74 became demented during the incident period. This figure differs slightly from our original report' ${ }^{\prime}$ because of subsequent additional reviews and telephone calls. The table summarizes demographic and disease features, ascertained during the prevalence period, for patients who did and did not become demented during the incidence period.

The items from the table that met our minimal criteria for differentiating the incident and nonincident dementia patients were then forward loaded into a logistic regression model predicting incident dementia. Adverse effects to levodopa therapy (confusion or psychosis) were combined for this analysis. Independent and significant predictors of incident dementia included masked facies at disease onset (odds ratio $[\mathrm{OR}]=6.1 ; 95 \% \mathrm{CI}=1.4$ to 26.9 ) and the following disease features, observed at the prevalence visit: depression ( $\mathrm{OR}=2.9 ; 1.5$ to 6.0 ), confusion or psychosis on levodopa $(\mathrm{OR}=3.3 ; 1.3$ to 8.7 ), older age ( $\leq 69$ versus $>69$ years; OR $=2.7$; 1.4 to 5.5 ), and higher CUPDRS scores ( $\leq 24$ versus 
$>24 ; \mathrm{OR}=3.0 ; 1.5$ to 6.2 ).

Since follow-up time was variable across patients and the onset of dementia occurred at variable times during follow-up, we utilized a stepwise Cox proportional hazards model with forward loading to extend and verify the results of the logistic regression. The Cox analysis essentially controlled for age, since follow-up time was referenced to the patient's age. The results of this analysis were identical, with depression, higher CUPDRS scores, confusion or psychosis on levodopa, and masked facies at disease onset each independent and significant predictors of incident dementia ( $p<$ 0.001 for each).

Discussion. There is little existing information about antecedent clinical features that predict dementia in PD. In a retrospective study, Marder et $\mathrm{al}^{4}$ found that first-degree relatives of demented patients with PD had at least six times the risk of dementia than those of nondemented patients with PD. In a case control study, Salganik and Korczyn ${ }^{5}$ reported increased risk of dementia in PD associated with previous exposure to the Holocaust, cardiovascular abnormalities, and possibly lower socioeconomic and educational levels. Biggins et $\mathrm{al}^{3}$ found that incident PD dementia was associated with older age at disease onset, longer disease duration, and older age at inclusion into their study.

Our present findings complement, in part, the associations with dementia noted in our prevalence study utilizing this cohort. ${ }^{6}$ Prevalent dementia was associated with advanced age, more severe disease, and adverse responses to levodopa therapy. In the present analyses, these represent antecedent clinical features for dementia.

There are several interpretations or inferences that might be drawn from the association of depression with incident dementia. We previously reported that $\mathrm{PD}$ patients with major depression have lower levels of CSF 5-HIAA than nondepressed patients, suggesting that the serotonergic system may contribute to susceptibility to depression in PD. ${ }^{14}$ We also found reduced CSF 5-HIAA levels in demented parkinsonians, with a compounding of effects in patients with both depression and dementia. ${ }^{15}$ These observations suggest some overlap in the mechanisms that contribute to both depression and dementia in PD. A second possibility is that the early signs of dementia might often mimic depression or that depression and dementia often occur concomitantly in PD.

Several issues affecting interpretation of this study must be addressed. Neuropsychological testing was not used, and diagnosis was based on chart review and telephone contacts. Both of these factors could lead to some misclassification, and per- haps undercounting of dementia. However, the issues should be nondifferential with regard to $t$ question of determining antecedent clinical fe tures. Our sample comprised only patients $w$ came to medical attention at our institution a may not be representative of the typical patie with PD. There is the possibility of selection bi since our center's movement disorder experti may have attracted unusual patients. However, $t$. antecedent clinical features considered here a typical features of $P D$ and there is no reason believe that they should be differentially affect by the presence of unusual patients.

\section{References}

1. Mayeux R, Chen J, Mirabello E, et al. An estimate of $t$ incidence of dementia in idiopathic Parkinson's diseas Neurology 1990;40:1513-1517.

2. Rajput AH, Offord KP, Beard CM, Kurland LT. A case-co trol study of smoking habits, dementia, and other illness. in idiopathic Parkinson's disease. Neurology 1987;37:22 232.

3. Biggins CA, Boyd JL, Harrop FM, et al. A controlled, long tudinal study of dementia in Parkinson's disease. J NeurNeurosurg Psychiatry 1992;55:566-571.

4. Marder K, Flood P, Cote L, Mayeux R. An pilot study of ris factors for dementia in Parkinson's disease. Mov Disor 1990;5:156-161.

5. Salganik I, Korczyn A. Risk factors for dementia i Parkinson's disease. Adv Neurol 1990;53:343-347.

6. Mayeux R, Stern Y, Rosenstein R, et al. The prevalence dementia in idiopathic Parkinson's disease. Arch Neurc 1988;45:260-262.

7. Marder K, Leung D, Tang M, et al. Are demented patient with Parkinson's disease accurately reflected in prevalenc surveys? A survival analysis. Neurology 1991;41:1240-1243.

8. Ward CD, Gibb WR. Research diagnostic criteria fo Parkinson's disease. In: Streifler MB, Korczyn AD, Melame. E, Youdim MBH, eds. Parkinson's disease: anatomy, pathol ogy and therapy. New York: Raven Press, 1990:245-249.

9. Hughes AJ, Daniel SE, Kilford L, Lees AJ. Accuracy of clini cal diagnosis of idiopathic Parkinson's discase: a clinico pathological study of 100 cases. J Neurol Neurosur\& Psychiatry 1992;55:181-184.

10. Hughes AJ, Ben-Schlomo Y, Daniel SE, Lees AJ. What fea. tures improve the accuracy of clinical diagnosis ir Parkinson's disease: a clinicopathologic study. Neurolog. 1992;42:1142-1146.

11. American Psychiatric Association. Diagnostic and statistical manual, 3rd ed. Washington, DC: APA, 1979.

12. Lesser RP, Fahn S, Snider SR, et al. Analysis of the clinical problems in parkinsonism and the complications of longterm levodopa therapy. Neurology 1979;29:1253-1260.

13. Schwab JF, England AC. Projection technique for evaluating surgery in Parkinson's disease. In: Gillinghan FS , Donaldson MN, eds. Third Symposium on Parkinson's Disease. Edinburgh, Scotland: E\&S Livingston, 1969:152157.

14. Mayeux R, Stern Y, Cote LJ, Williams JBW. Altered serotonin metabolism in depressed patients with Parkinson's disease. Neurology 1984;34:642-646.

15. Sano M, Stern Y, Cote L, et al. Co-existing dementia and depression in Parkinson's disease. Arch Neurol 1989;36:1284-1286. 